\title{
GaAlAs PHOTOTHERAPY FOR TENSION AND COMPRESSION RELATED TEMPOROMANDIBULAR DISORDERS
}

\author{
Vassil Svechtarov ${ }^{1}$, Savina Nencheva-Svechtarova ${ }^{1}$, Tsvetan Tonchev ${ }^{2}$, \\ Krassimira Prodanova ${ }^{3}$ \\ ${ }^{1}$ Department Oral and Maxillofacial Surgery, Faculty of Dental Medicine, \\ Medical University of Sofia \\ ${ }^{2}$ Department Oral and Maxillofacial Surgery and Special Dental Imaging, \\ Faculty of Dental Medicine, Medical University of Varna \\ ${ }^{3}$ Department Stochastics and Optimization, Faculty of Applied Mathematics and \\ Informatics, Technical University - Sofia
}

\begin{abstract}
Introduction: The aim of this study is to evaluate the distribution of the most common etiological factors responsible for the development of chronic temporomandibular disorders, and to test the pain - relief effectiveness of combined gallium-aluminum-arsenide laser (GaAlAs; $785 \mathrm{~nm}$ ) and superluminiscent diods (SLD; $633 \mathrm{~nm}$ ) phototherapy (MedX 1100 device) for most common tension and compression pain-related diagnostic subgroups of chronic temporomandibular disorders.
\end{abstract}

Material and methods: This study includes 62 patients - 7 men and 55 women, aged between 21-74 years with clinical signs and symptoms of chronic temporomandibular joint and myofascial pain. Using DC/TMD instruments the most common causative factors were identified, and part of the TMDs assessed were classified to tension and compression related diagnostic subgroups. Trigger point oriented combination of laser (MedX 1100 console with three GaAlAs laser diodes, $785 \mathrm{~nm}, 3 \times 33 \mathrm{~mW}, 100 \mathrm{~s}, 8 \mathrm{~J} / \mathrm{cm} 2$ per spot) and SLD red light sessions $(633 \mathrm{~nm}, 200 \mathrm{~mW}, 300 \mathrm{~s}, 8 \mathrm{~J} / \mathrm{cm} 2$ per spot) was applied for temporomandibular joints and affected muscles.

Results and conclusion: Using descriptive analysis and paired samples Student's t-test, the most statistically manifested pain reduction is found for Arthralgia attributed to Tension-related TMDs (Subluxations, Dislocations, Sprain and strain of joints, ligaments and TMJ - related structures, Flexion-extension injuries; $p=0,000001)$, followed by Myalgia for the same disorders $(p=0,000014)$, followed by Arthralgia attributed to Compression-related TMDs (bruxism, psychosomatic parafunctions, occlusal microtrauma, dentofacial anomalies and malocclusions, $p=0,000037)$, and Myalgia attributed to the same TMDs $(p=0,000808)$. This study shows that combined phototherapy provides

Address for correspondence:

Vassil Svechtarov

Department Oral and Maxillofacial Surgery,

Faculty of Dental Medicine,

Medical University of Sofia

1 Sv.Sv. Georgi Sofiyski Blvd.

1431 Sofia

e-mail:vassilsvechtarov@yahoo.com

Received: July 27, 2015

Accepted: December 1, 2015 statistically significant reduction of pain symptoms for the most common temporomandibular disorders, and that Arthralgia and Myalgia attributed to tension-related disorders are better influenced by combined phototherapy than compression-related ones.

Keywords: gallium-aluminum-arsenide laser (GaAlAs), temporomandibular disorders (TMD), diagnostic criteria (DC/TMD) 
Vassil Svechtarov, Savina Nencheva-Svechtarova, Tsvetan Tonchev et al.

\section{INTRODUCTION}

Temporomandibular disorders (TMD) is a collective term applied for all pathologic conditions involving the masticatory muscles, the temporomandibular joint (TMJ) and all functionally related orofacial structures. They are characterized with a specific pain symptoms and dysfunctions, affecting up to $12 \%$ of the population of the developed countries $(20,21,22)$. Chronification is observed in $5-15 \%$ of all patients, with symptoms that are present for more than six months and the pain is persistent or remittent $(1,9)$. Patients with prolonged pain symptoms are a difficulty to treat and influence by most of the known methods.

The diagnostic of these disorders was always a subject of many discussions, but in 2014 after the realization of the Validation Project and TMJ Impact Project of the National Institute for Dental and Craniofacial Research (NIDCR) and International Association for Dental Research (IADR), a new taxonomy was developed, that is based on the definitions of the American Academy of Orofacial Pain (AAOP). Recommendations on new diagnostic criteria and the appropriate tools were also adopted (Diagnostic Criteria for Temporomandibular Disorders, DC/ TMD), which are modifications of the Research Diagnostic Criteria for TMD (RDC/TMD) from 1992. $(20,22,25)$ The American Association for Orofacial Pain (AAOP) has included 12 most common painrelated TMDs in a new revision of their Guidelines manual such that the DC/TMD and the AAOP taxonomic system for TMDs are now consistent (20). The DC/TMD are synchronized with the nosology of the Tenth Revision of the International Classification of Diseases (ICD-10). The criteria according to DC/ TMD Axis I have sensibility $\geq 0.86$, specificity $\geq 0.98$, and inter-examiner reliability $\geq 0.85$ (22).

A specific feature of this diagnostic system is that the majority of cases require multiple coding of the disorder. For example, a complicated case of osteoarthritis with internal derangements and myofascial complaints which usually have multiple signs and symptoms, is coded as arthralgia+ degenerative disorder+ type of disc displacement+ type of myalgia (22). This approach refines the diagnostics, and brings specificity of all structures involved. However, in ICD-10 there are summarized diagnoses like
K07.6 Temporomandibular joint-pain-dysfunction syndrome, as well as etiologically based diagnoses like S03 Dislocation, sprain and strain of joints and ligaments of head, which are more useful in everyday practice (27).

Schiffman et al. (22) define as a strategic goal for the refinement of the contemporary diagnostic protocols, the development of diagnostic criteria based on the mechanisms and etiology of the TMD. According to Dworkin the correct diagnosis should be based on the knowledge about the etiology and pathophysiology of the disease. A known pathophysiology provides the identification of an etiologic agent and the description of the pathogenetic mechanism leading to the onset of the disease and to its natural course. The biopsychosocial model for TMD, which is still considered the best-fitting model for TMD assessment, has to be taken into full account when reporting findings of any kind of investigations in the field of TMD and orofacial pain (15).

In contrary to the similarity of the symptoms, Greene points out that a specific etiopathogenesis is rarely demonstrable, since most cases seem to have a multifactorial etiopathogenetic pathway (15). The complex etiopathogenesis and the variability of symptoms makes it difficult to adopt standardized diagnostic and therapeutic protocols. The limited knowledge about the TMD etiology prevents clinicians from pursuing a causative therapy and to define a successful treatment on the basis of the eradication of the causal factor.

Following the logic of the TMD classification to common and rare, the etiological factors can also be divided according to this scheme. Orlova et al. (19) found that approximately $50 \%$ of the patients associate the beginning of the TMD with surgical or dental trauma when the procedure took longer than an hour. The myofascial pain symptoms developed 1012 days after the procedure with periodic pains and/ or muscle stiffness. The trauma is a single act and the pain reactivation afterwards may be due to other circumstances. The trauma to the cervical part of the vertebrae and the whiplash trauma may initiate temporomandibular symptoms in $10 \%$ of the cases. In $40 \%$ of the patients with TMD the diagnosis includes stress, anxiety, depression and psychosomatic induced parafunctions; the intensity of the pain in

Scripta Scientifica Medicinae Dentalis, vol. 1, №2, 2015, pp. 24-29 
such cases increases in the next 2-3 months after its initiation. For that group of patients is characteristic the remittent type of pain, it periodically disappears but under the influence of provoking factors it reappears again. As time passes by the periods of remission grow shorter and gradually the pain becomes chronic.

The disorders caused by the action of abnormal tension or compression forces may be divided into two main subgroups - tension related (TR) and compression related (CR). Tension type forces act in luxations, subluxations, iatrogenic strains, flexion-extension type cervical traumas, etc. Abnormal compression forces are found in bruxism, stress and behavioral - induced parafunctions, chronic occlusal trauma, orthodontic anomalies such as distal class II occlusion, distal crossbite, muscular hypertrophy etc. The clinical practice also indicates the need of pathophysiologically - based disorder subgrouping; for example, the standard use of occlusal splints has mainly decompression effect on the intra articular structures. On the contrary, the hypermobility and dislocations demand partial or complete immobilization of the mandible. These are totally opposite procedures for treatment of the same disorder due to the difference in the causative factors. A typical example are the disk displacements which can be found in mandibular hypermobility, but can also be caused by a chronic compression of the articular disk in bruxism.

For symptomatic treatment of the pain in patients with TMD can be used lasers and super luminous diodes (SLD). The energy they release provides temporary increase of the microcirculation, temporary pain relief, relaxation of the musculature and muscle spasms overcome. The most commonly used laser is the GaAlAs laser operating in the diapason of $780-870 \mathrm{~nm}(3,5,6,7,16,26)$. Brosseau et al. (4) report for a short relief of the morning pain and stiffness after low level laser therapy in patients with rheumatism, while for patients with osteoarthritis there is not enough data. Concerning myofascial pain Laakso et al. (13) support the positive therapeutic effect of the lasers with $670 \mathrm{~nm}, 10 \mathrm{~mW}$ and $820 \mathrm{~nm}, 25 \mathrm{~mW}$, accounting better effect with $820 \mathrm{~nm}$. Fikachkova et al. $(10,11)$ treat 61 patients with GaAlAs laser using doses of 10 and $15 \mathrm{~J} / \mathrm{cm}^{2}, 400 \mathrm{~mW}$ power output, 830 $\mathrm{nm}$ wave length, 10 procedures, and report a positive pain reducing effect especially in chronic cases. Baxter et al. (2) offer energy density of 8 to $12 \mathrm{~J} / \mathrm{cm}^{2}$ for the mandibular joint. Maia et al. (14) perform a systematic analysis of 14 articles on the use of LLLT in the treatment of TMD, in thirteen of them a positive effect on the reduction of pain is observed. According to a meta analysis of Chang et al. (8) the analgesic effect achieved with laser therapy with a wave length of 830 and $780 \mathrm{~nm}$ is moderate to superior.

Publications regarding the application of combined infrared and red light phototherapy of patients with chronic temporomandibular disorders and myofascial pain are basically missing. Former research have reviewed the subject on single laser therapy for definite muscle groups or TMJ. Up till now the combined cold laser therapy has been researched in the context of seven of the DC/TMD diagnosis with high specificity and sensibility $(17,18,24)$. However, from a clinical point of view the evaluation of any kind of treatment of bigger diagnostic subgroups that are based on etiopathogenetic factors involved is very expedient and in a total agreement with DC/ TMD methodology.

\section{AIM OF THE STUDY}

The aim of this study is to evaluate the distribution of the most common etiological factors responsible for the development of chronic temporomandibular disorders, and to test the pain - relief effectiveness of combined gallium-aluminum-arsenide laser (GaAlAs; $785 \mathrm{~nm}$ ) and superluminiscent diods (SLD; $633 \mathrm{~nm}$ ) phototherapy (MedX 1100 device) for most common tension and compression - related diagnostic subgroups in 62 individuals with chronic temporomandibular disorders.

\section{MATERIAL AND METHODS}

This study includes 62 patients - 7 men and 55 women, aged between 21-74 years (avg. - 42,77; Std Dev - 13,94). All cases had clinical signs and symptoms of chronic joint and / or myofascial pain. For the purpose of the study were selected the etiology - related questions from DC/TMD: Symptom Questionnaire, Graded Chronic Pain Scale (v2), Patient Health Questionnarie-9, GAD-7, Oral Behaviors Checklist - for the presence of bruxism, stress induced parafunctions; other specific questions like the presence of iatrogenic trauma, etc. (17). 
Vassil Svechtarov, Savina Nencheva-Svechtarova, Tsvetan Tonchev et al.

Trigger point oriented combination of laser (MedX 1100 console with three GaAlAs laser diodes, $785 \mathrm{~nm}, 3 \times 33 \mathrm{~mW}, 100 \mathrm{~s}, 8 \mathrm{~J} / \mathrm{cm}^{2}$ per spot) and SLD red light sessions $\left(633 \mathrm{~nm}, 200 \mathrm{~mW}, 300 \mathrm{~s}, 8 \mathrm{~J} / \mathrm{cm}^{2}\right.$ per spot) was applied for temporomandibular joints and affected muscles. The outcome measurements for pain intensity before and after treatment included visual analogue scale (VAS) scores. The pain intensity measurements were recorded separately for the joints and muscles before and after the completion of six sessions ( 3 times per week) of photo therapy. A descriptive analysis for the causative factors and a Student's t-tests of paired samples for both tension - related and compression - related subgroups were used for statistical analysis with STATISTICA software.

\section{RESULTS}

1. Descriptive statistics. The overall percent of the disorders caused by hypermobility, iatrogenic strains and cervical trauma (ICD-10, S03 - Dislocation, sprain and strain of joints and ligaments of head, S03- subluxation, Dislocation of jaw, S13.4 - Sprain and strain of cervical spine, Flexion-extension injury, S03.4, - iatrogenic Sprain and strain of jaw and S03.5- of other orofacial structures) is highest of all studied factors $-58,05 \%$ in total. From them a large percentage is due to iatrogenic strains $(24,19 \%)$, which should focus the attention of the clinicians on the length of the dental procedures and the surgical interventions in the oral cavity, because these can cause permanent organic damage of the joint structures and formation of latent myofascial trigger points. The total percentage of bruxism (F45.8), muscle fatigue from behavioral and psychologic (stress, anxiety, depression) parafunctions (Psychosomatic disorders F45.9) is 35,47\%, which comes to prove the importance of the chronic compressive hyper functionally-dependent micro trauma. Dentofacial anomalies and malocclusions (K07.1K07.4) consist $19,34 \%$ of the causative factors, the degenerative joint disease (M19.0 - M19.2) and systemic arthritides (rheumatoid arthritis, M06.9) - 22,57\%, and the group of the more uncommon causes such as posture provoked myalgia, temperature provoked myalgia (M79.1), dysphagia, (R13), neurologically related dystonia or dyskinesia is $16,12 \%$. The total percentage of the factors is above 100, because it is typ-

Table 1. T-test for Dependent Samples for pain reduction for Tension-related and Compression-related TMDs

\begin{tabular}{|c|c|c|c|c|c|c|c|}
\hline TMD & $\begin{array}{l}\text { Pain inten- } \\
\text { sity mean } \\
\text { VAS scores }\end{array}$ & $\mathrm{SD}$ & $\mathrm{N}$ & $\mathrm{T}$ & $\mathrm{P}$ & $\begin{array}{l}\text { Conf. } \\
-95,00 \%\end{array}$ & $\begin{array}{l}\text { Conf.+ } \\
95,00 \%\end{array}$ \\
\hline $\begin{array}{l}\text { Arthralgia attributed to Tension- } \\
\text { related TMDs before treatment }\end{array}$ & 3,193 & 3,253 & & & & & \\
\hline $\begin{array}{l}\text { Arthralgia attributed to Tension- } \\
\text { related TMDs after treatment }\end{array}$ & 0,612 & 1,121 & 62 & 7,42 & 0,000001 & 1,885 & 3,275 \\
\hline $\begin{array}{l}\text { Myalgia attributed to Tension-related } \\
\text { TMDs before treatment }\end{array}$ & 2,274 & 3,811 & & & & & \\
\hline $\begin{array}{l}\text { Myalgia attributed to Tension-related } \\
\text { TMDs after treatment }\end{array}$ & 0,338 & 1,447 & 62 & 4,72 & 0,000014 & 1,116 & 2,754 \\
\hline $\begin{array}{l}\text { Arthralgia attributed to Compression- } \\
\text { related TMDs before treatment }\end{array}$ & 1,741 & 3,061 & & & & & \\
\hline $\begin{array}{l}\text { Arthralgia attributed to Compression- } \\
\text { related TMDs after treatment }\end{array}$ & 0,306 & 0,667 & 62 & 4,45 & 0,000037 & 0,790 & 2,080 \\
\hline $\begin{array}{l}\text { Myalgia attributed to Compression-re- } \\
\text { lated TMDs before treatment }\end{array}$ & 1,838 & 4,113 & & & & & \\
\hline $\begin{array}{l}\text { Myalgia attributed to Compression- } \\
\text { related TMDs after treatment }\end{array}$ & 0,290 & 0,894 & 62 & 3,52 & 0,000808 & 0,670 & 2,426 \\
\hline
\end{tabular}

Scripta Scientifica Medicinae Dentalis, vol. 1, №2, 2015, pp. 24-29 
ical for these disorders that there is more than one factor that the patients report.

2. T-test for Dependent Samples. The most statistically manifested pain reduction is found for Arthralgia attributed to Tension-related TMDs (subluxations, dislocations, sprain and strain of joints, ligaments and other TMJ - related structures, flexion-extension injuries; $\mathrm{p}=0,000001$ ), followed by Myalgia attributed to the same TMDs ( $\mathrm{p}=0,000014$ ), followed by Arthralgia attributed to Compression-related TMDs (bruxism, psychosomatic parafunctions, occlusal micro trauma and malocclusions, $\mathrm{p}=0,000037$ ), and Myalgia attributed to the same disorders $(\mathrm{p}=0,000808)$. The data proved that Arthralgia and Myalgia attributed to tension-related disorders are better influenced by combined phototherapy than compression-related ones.

The present study established that the combined phototherapy is an effective pain reducing mono-therapy in $96,77 \%$ of the cases. From the whole group only in two of the cases there was not therapeutic effect observed. The first case is of a 44 years old woman with manifest bilateral subluxation, flattening of the condyles, anterior disk displacement without reduction and limited opening $(33 \mathrm{~mm})$, myofascial pain in the temporal muscle, the insertions of the medial pterygoid muscle and masseter unilaterally, with referred pain towards the pharynx and the molar area of the mandible. The second case is of a 36 years old male patient with bilateral subluxation, spasms of the lateral pterygoid muscle unilaterally with referred pain towards the maxillary sinus and the upper molars. These single cases come to prove that in some hypermobile conditions single laser therapy does not provide the desired effect and has to be combined with medications and other physiotherapeutic and/ or orthopedic therapies.

\section{DISCUSSION}

This study shows that GaAlAs photo therapy provides statistically significant reduction of pain symptoms in the most common pain-related temporomandibular disorders. Subgrouping according to the most common causative factors that have similar pathophysiological mechanism assists the prognosis of the treatment. Pain assessment is the target of all studies on treatment effectiveness. The best outcome measure for clinical trials on TMD patients is the Visual Analogue Scale (VAS) which is broadly employed and valid method to assess pain in clinical conditions. Fifteen percent decrease in VAS ratings was interpreted by subjects as being insignificant. The fluctuant nature of the symptoms and the high rate of spontaneous remission characterizing myofascial pain lead to a rate of improvement of about $26 \%$ in non-treated patients. Clinically significant improvement is a $40 \%$ decrease in VAS ratings (15). In the present study the mean reduction of pain intensity is $83.11 \%$ (SD 1,90), which is a result comparative to the studies of Simonovic et al. (23) on 243 patients, in which the pain is reduced with more than $70 \%$ following mono laser therapy with wavelength of $820 \mathrm{~nm}$, very close to the wavelength used in this study - $785 \mathrm{~nm}$.

\section{CONCLUSION}

The findings about combined phototherapy pain reduction for TMD suggest that it may be of a great benefit to the clinicians for the symptomatic treatment of these complex cases. The effectiveness of the combined laser- SLD therapy in both the tension- and compression-related TMD groups is high. There are single cases in which there is no treatment effect observed. In most of the cases using a total dose of up to $30 \mathrm{~J}$ per visit, the therapeutic course rarely exceeds more than six visits which is a total of two - three weeks.

\section{REFERENCES}

1. American Association of Oral and Maxillofacial Surgeons. Parameters of Care: Clinical Practice Guidelines for Oral and Maxillofacial Surgery (AAOMS ParCare 2012). Journal of Oral and Maxillofacial Surgery. 2012;70:204-232.

2. Baxter G, C Diamantopoulos, S O'Kane et al. Therapeutic Lasers Theory and Practice., New York: Churchill Livingstone; 1997. 208-209

3. Bjordal J, C Couppe, R Chow et al. A systematic review of low level laser therapy with locationspecific doses for pain from chronic joint disorders. The Australian Journal of Physiotherapy. 2003;49:107-116.

4. Brosseau L, V Welch, G Wells et al. Low level laser therapy for osteoarthritis and rheumatoid arthritis: a meta-analysis. Journal of Rheumatology. 2000;27:1961-1969. 
Vassil Svechtarov, Savina Nencheva-Svechtarova, Tsvetan Tonchev et al.

5. Carrasco T, L Gnerisol, D Gnerisol et al. Evaluation of low intensity laser therapy in myofascial pain syndrome. Cranio, 2009;27:243-247.

6. Carrasco T, M Mazzetto, R Mazzetto et al. Low intensity laser therapy in temporomandibular disorder: a phase II double-blind study. Cranio. 2008;26:274-81.

7. Cetiner S, S Kahraman, S Yucetas. Evaluation of low-level laser therapy in the treatment of temporomandibular disorders. Photomed Laser Surg. 2006;24:637-641.

8. Chang W, C Lee, H Lin et al. A Meta-analysis of Clinical Effects of Low-level Laser Therapy on Temporomandibular Joint Pain. J Phys Ther Sci. 2014;26:1297-1300.

9. Diagnostic Criteria for Temporomandibular Disorders (2014), Complete DC/TMD Instrument Set, International RDC/TMD Consortium, http://www. rdc-tmdinternational.org/TMDAssessmentDiagnosis/DCTMD.aspx

10. Fikackova H, T Dostalova, R Vosicka et al. Arthralgia of the temporomandibular joint and low-level laser therapy. Photomedicine and Laser Surgery. 2006;24:522-527.

11. Fikackova H, T Dostalova, L Navratil et al. Effectiveness of low-level laser therapy in temporomandibular joint disorders: a placebo-controlled study. Photomedicine and Laser Surgery. 2007;25:297-303.

12. Huang GJ, LeResche L, Critchlow CW, Martin MD, Drangsholt MT. Risk factors for diagnostic subgroups of painful TMD. JDR. 2002;81(4):282-8.

13. Laakso EL, C Richardson, T Cramond. Pain scores and side effect in response to low level laser therapy for myofascial trigger points. Laser Ther. 1997;9:67-72.

14. Maia M, L Bonjardin, S Quintans et al. Effect of low-level laser therapy on pain levels in patients with temporomandibular disorders: a systematic review. J Appl Oral Sci. 2012;20:594-602.

15. Manfredini D, Arboretti R, Guarda - Nardini L, Carrozzo E, Salmaso L. Statistical approaches to orofacial pain and temporomandibular disorders research. Springer; 2014.

16. Mazzetto M,T Carrasco,E Bidinelo et al. Low intensity laser application in temporomandibular disorders: a phase I double-blind study. Cranio. 2007;25:186-192.
17. Nencheva-Svechtarova S, Svechtarov V, Gisbrecht A, Uzunov Tz. Clinical and experimental study of GaAlAs phototherapy for temporomandibular disorders. Acta Medica Bulgarica. 2014;41(2):49-54.

18. Nencheva-Svechtarova S, Svechtarov V, Uzunov Tz, Prodanova K. Effectiveness of GaAlAs phototherapy according to diagnostic driteria for temporomandibular disorders (DC/TMD). Acta Medica Bulgarica. Forthcoming. 2015.

19. Orlova O, L Mingizova, A Wein. Myofascial facial pain syndrome: new aspects of clinics, pathogenesis and treatment. New In Sstomatology. 2003;1(109):1-5.

20. Peck C, J Goulet, F Lobezzoo et al. Expanding the taxonomy of the diagnostic criteria for temporomandibular disorders. Journal of Oral Rehabilitation. 2014;41:2-23.

21. Scrivani S, D Keith, L Kaban. Temporomandibular Disorders. N Engl J Med. 2008;359:2693-2705.

22. Schiffman E, R Ohrbach, E Truelove et al. Diagnostic Criteria for Temporomandibular Disorders (DC/ TMD) for Clinical and Research Applications: recommendations of the International RDC/ TMD Consortium Network and Orofacial Pain Special Interest Group. J Oral Facial Pain Headache. 2014;28:6-27.

23. Simonovic Z. LLLT with trigger points technique: a clinical study on 243 patients. Journal of Clinical Laser Medicine and Surgery. 1996;14:163-167.

24. Svechtarov V, Nencheva-Svechtarova S, Uzunov Tz. Analysis of chronic temporomandibular disorders based on the latest diagnostic criteria. Acta Medica Bulgarica, 2015;42(1):49-55.

25. The American Academy of Orofacial Pain. Orofacial pain: guidelines for assessment, diagnosis and management, 5th ed. Chicago (IL): Quintessence Publishing Co, Inc.; 2013.

26. Venancio R, CM Camparis, R Lizarelli. Low intensity laser therapy in the treatment of temporomandibular disorders: a double-blind study. J Oral Rehabil. 2005;32:800-807.

27. World Health Organization. ICD-10 Version: 2015, apps.who.int/classifications/icd10/

Scripta Scientifica Medicinae Dentalis, vol. 1, №2, 2015, pp. 24-29 